\title{
Posttraumatic endophthalmitis due to tobacco drying wire treated with vitrectomy, temporary keratoprosthesis, and keratoplasty
}

\author{
Małgorzata Ozimek ${ }^{1}$, Katarzyna Nowomiejska ${ }^{1}$, Cesare Forlini' ${ }^{2}$, Robert Rejdak ${ }^{1,3}$ \\ 'Department of General Ophthalmology, Medical University of Lublin, Lublin, Poland \\ ${ }^{2}$ Department of Ophthalmology, Santa Maria Delle Croci Hospital, Ravenna, Italy \\ ${ }^{3}$ Department of Experimental Pharmacology, Medical Research Centre, Polish Academy of Sciences, Warsaw, Poland
}

\begin{abstract}
AIM. To report a case of posttraumatic endophthalmitis with corneal opacity that was treated by combined penetrating keratoplasty using temporary keratoprosthesis and pars plana vitrectomy (PPV) with silicon oil tamponade. CASE PRESENTATION. A 35-year-old woman was admitted to our clinic after open globe injury due to wire used for tobacco drying. At the time of her first visit, her best-corrected visual acuity was light perception. On the day of admission a 20-G PPV with phacoemulsification and vancomycin administration in tapping was performed. The operation was aborted due to visualisation constraints. Four days later 20-G PPV in combination with penetrating keratoplasty with use of temporary keratoprosthesis and silicon oil tamponade was performed. General and topical antibiotics and topical antifungal drugs were administered. Five months after hospitalisation visual acuity was light perception, and the corneal graft remained clear, but proliferative vitreoretinopathy was observed.

CONCLUSIONS. Combined penetrating keratoplasty using temporary keratoprosthesis and PPV could be advantageous in managing visualisation constraints due to posttraumatic endophthalmitis; however, functional results are not satisfactory.
\end{abstract}

KEY WORDS: penetrating injury, endophthalmitis, vitrectomy, temporary keratoprosthesis, penetrating keratoplasty

Ophthalmol J 2016; Vol. 1, No. 1, 36-39

\section{INTRODUCTION}

Endophthalmitis is a severe complication of open globe injury. About $25 \%$ of endophthalmitis cases are a result of ocular trauma, and $7 \%$ of all open globe injuries develop endophthalmitis [1]. Risk factors for developing endophthalmitis after acute eye injury include lens capsule rupture, dirty wound, advanced age of the patient, presence of intraocular foreign bodies, and initial presentation more than 24 hours after injury $[2,3]$. The most common pathogens that cause posttraumatic endophthalmitis are Bacillus and Streptococcus species. Bacillus species are associated with more aggressive infection and are especially common in intraocular foreign bodies with organic composition [4]. Therefore, eye injuries in which there is a contamination of the wound by organic material have worse prognosis. Other, less common pathogens are: S. epidermidis, S. aureus, Propionibacterium acnes, Pseudomonas, Gram-negative organisms, fungi, and mixed pathogens [5]. It is essential to take immediate treatment, both surgical and pharmacological in order to achieve the best outcomes. We present a case report of posttraumatic endophthalmitis with corneal opacity, and the treatment that we provided. 


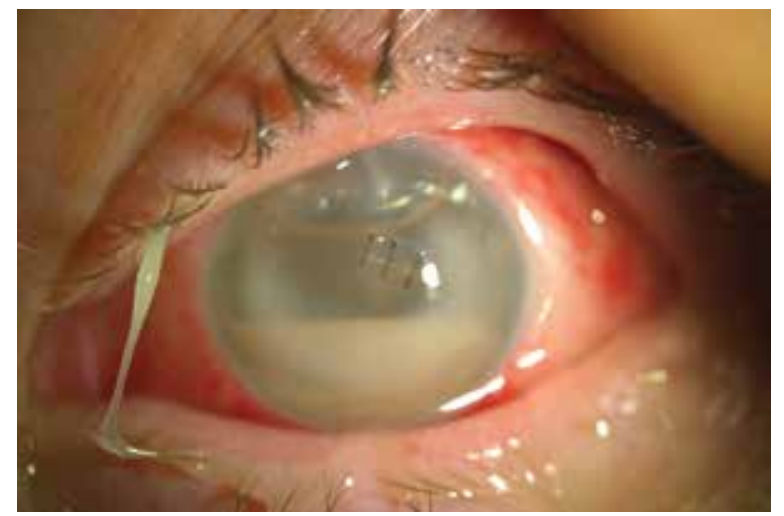

FIGURE 1. The eye condition on the date of admission

\section{CASE PRESENTATION}

A 35-year-old woman in good general health was referred to our clinic with suspicion of endophthalmitis with suprachoroidal and intravitreal haemorrhage of the left eye, two days after open globe eye injury due to the wire used for tobacco drying. The patient hurt herself while working on a tobacco plantation. The corneal penetrating wound was sutured during an emergency procedure in another hospital.

At the time of patient admission to our clinic best-corrected visual acuity was 1.0 in the right eye and light perception in the left eye. The intraocular pressure in both eyes was within the normal range. Slit lamp examination revealed severe hyperaemia of the conjunctiva, chemosis, opacity and oedema of the cornea, and hypopyon in the anterior chamber. Fluid leakage from corneal wound was observed (Fig. 1). The fundus of the left eye was invisible due to corneal opacity, but B-mode ultrasonography showed dense opacities in vitreous body with oedema of the retino-choroidal layer (Fig. 2). In the right eye, there were no abnormalities and, importantly, no symptoms of sympathetic ophthalmia. There were no signs of foreign bodies in orbit X-ray. Acute posttraumatic endophthalmitis was diagnosed.

On the day of admission to our clinic a $20-G$ three-port pars plana vitrectomy (PPV) with phacoemulsification and vancomycin administration was performed. The operation was abandon due to visualisation constraints. The vitreous obtained during the surgery was sent to the laboratory for microbiological tests. The patient was treated empirically with intravenous cefazolin and topical moxifloxacin and natamycin. Microbiological tests revealed Staphylococcus epidermidis and Bacillus sp. sensitive to current treatment. Four days later 20-G PPV in combination with penetrating keratoplasty with use of temporary keratoprosthesis and

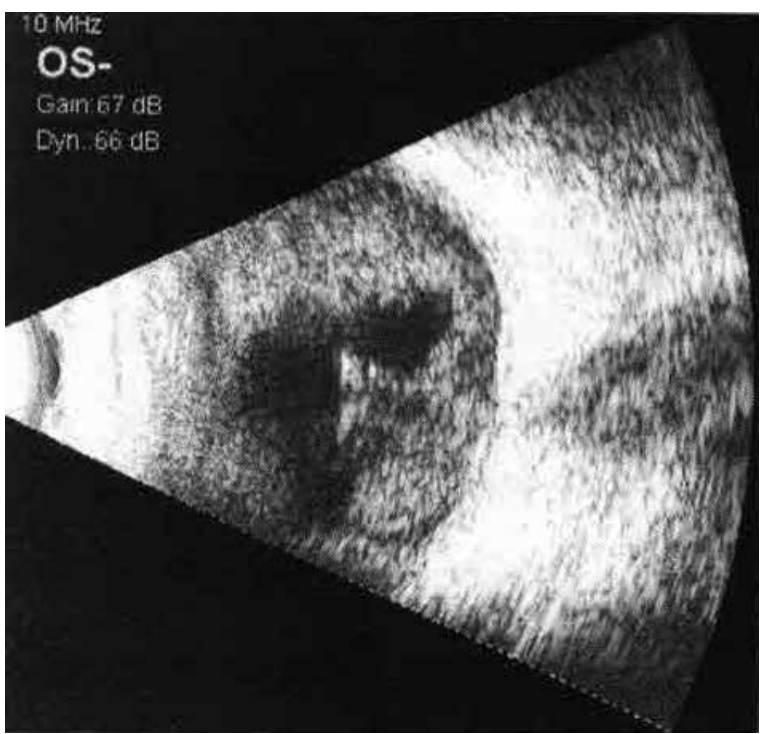

FIGURE 2. Dense opacities in vitreous body seen in ultrasonography

silicon oil tamponade was performed. The surgery was performed in general anaesthesia. First, three $20-\mathrm{G}$ sclerotomy ports were inserted at the pars plana $3.5 \mathrm{~mm}$ posterior to the limbus and sutured to the sclera. The opaque central portion of the cornea was excised with a corneal trephine. A Landers temporal keratoprosthesis (KTP) (Ocular Instruments, Bellevue, WA, USA) was sutured onto the recipient cornea with six single 9-0 nylon sutures. Standard PPV was then performed, using vancomycin in the irrigating solution. It was completed with detachment of posterior vitreous cortex and the vitreous skirt shaved in the periphery. We used the silicon oil tamponade because of the bad condition of the retina. During the vitrectomy, we saw severe vitreous opacification, a thick inflammatory membrane that covered the entire retina, and suprachoroidal and intravitreal haemorrhage. After completing the vitrectomy the TKP was removed and replaced with a donor corneal graft. The donor cornea was sutured onto the recipient cornea with 16 interrupted 10-0 nylon sutures. All corneal suture knots were buried. No intraoperative or postoperative complications were encountered. After the second surgery the patient additionally received topical dexamethasone six times a day and oral prednisone $50 \mathrm{mg}$ per day to prevent graft rejection. The study of subsequent aqueous humour samples showed no bacterial growth.

At five-month follow-up best-corrected visual acuity in the left eye was light perception on the left side, and the cornea graft remained clear, but prolif- 


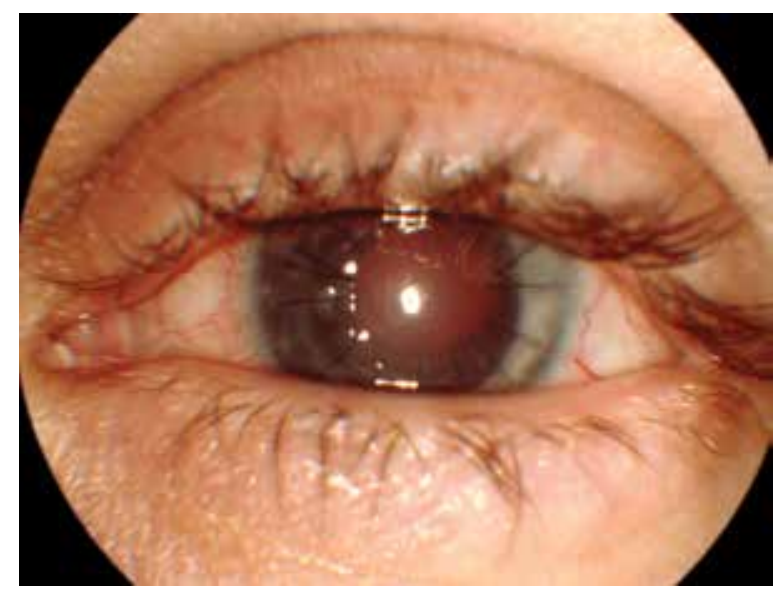

FIGURE 3. The eye after five months of the follow-up after combined surgery, clear corneal graft

erative vitreoretinopathy with retinal detachment in the temporal quadrant was observed (Fig. 3). Further operative treatment has not been undertaken. The fellow eye had no symptoms of sympathetic ophthalmia.

\section{DISCUSSION}

Development of endophthalmitis after trauma significantly reduces the chances of obtaining final functional vision. Immediate treatment is essential to achieve the best possible outcome. In the case of post-traumatic endophthalmitis bidirectional treatment is necessary: pharmacological and surgical. Recently, there has been a trend for early vitrectomy approach. Endophthalmitis, especially after trauma, is one of the biggest challenges for ophthalmologic surgeons. Opacification and oedema of the cornea, inflammatory material in the anterior chamber, non-transparent vitreous, and fragile, necrotic retina make the surgery extremely difficult. The advantage of PPV is the ability to obtain samples for culture, administer the drugs directly, and clear out pathogens, toxins, inflammatory materials, and opacities. Vitrectomy is done as a primary procedure, especially when endophthalmitis is associated with an intraocular foreign body. The surgical goal is 'complete' three-port PPV with maximal removal of the infectious masses from the eye. Vitrectomy must be performed very carefully, with anterior-posterior progress. It should include posterior vitreous cortex detachment, vacuum of the macular surface, and peripheral vitreous shaving. In cases of post-traumatic endophthalmitis is not recommended to use a small diameter vitrectoms. Silicon oil has to be considered when there are tears, detachment, or progressive necrosis of the retina [6]. If the condition of the cornea makes visualisation of deeper structures impossible, vitrectomy with temporary keratoprosthesis could be performed. It is a complicated procedure; however, it allows us to avoid delay in performing surgery and associated complications. An alternative procedure is endoscope-guided vitrectomy followed by corneal transplantation [6]. Endoscopy requires less time and fewer procedures than temporary keratoprosthesis. It gives the ability to postpone keratoplasty, which is important because of the high risk of graft failure in the early period after injury. Moreover, it allows us to observe the peripheral part of the retina, vitreous base, pars plana, and pars plicata, without manipulation of the anterior chamber, as well as scleral depression, reducing the risk of intraoperative complications such as haemorrhage [7]. Unfortunately, the equipment for endoscope-guided vitrectomy is not available in most Polish ophthalmology centres.

Antibiotics are administered during surgery in the irrigating solution or/and as intravitreal injections. Unless culture results are available, empiric therapy to cover both gram-positive and gram-negative organisms should be used. Current antibiotic standard protocols for intravitreal application include the peptide antibiotic vancomycin $(1.0 \mathrm{mg} / 0.1 \mathrm{~mL})$ for Gram-positive coverage, in combination with the $\beta$-lactam antibiotic ceftazidime $(2.2 \mathrm{mg} / 0.1 \mathrm{~mL})$ for Gram-negative coverage. In patients sensitive to $\beta$-lactam drugs, amikacin $(400 \mu \mathrm{g} / 0.1 \mathrm{~mL})$, an aminoglycoside antibiotic, might be considered instead of ceftazidime $[6,8]$. However, it should be used cautiously because its retinal toxicity has been reported. Some authors suggest that clindamycin $(250 \mu \mathrm{g} / 0.1 \mathrm{~mL})$ in combination with ceftazidime should be used in cases with an intraocular foreign body [9] because it is effective on Bacillus species. A second course of intravitreal antibiotics should be considered if the patient does not appear to be improving 48 to 72 hours after the initial administration [8]. Topical and systemic therapy should be continued with the same antibiotics as used for intravitreal therapy. Most patients are responsive to empiric therapy; otherwise it should be modified according to the clinical response and the antibiotic sensitivity profile of the cultured organism. In the case of suspicion of fungal infection antifungal therapy should be administered (intravitreally and systemically: amphotericin, optionally voriconazole, topically: natamycin) [5]. 
There were many controversies regarding the correct timing of vitreoretinal intervention in endophthalmitis and open eye globe injuries. Recent reports have advocated early intervention.

\section{CONCLUSIONS}

We describe a case of posttraumatic endophthalmitis with suprachoroidal and intravitreal haemorrhage and corneal opacity, which was successfully treated using combined penetrating keratoplasty, temporary keratoprosthesis, and PPV with silicon oil tamponade. Use of temporary keratoprosthesis could be advantageous in dealing with visualisation constraints due to penetrating trauma and posttraumatic endophthalmitis. This combined surgery permits integration of the eyeball; however, longitudinal functional results are not satisfactory.

\section{REFERENCES}

1. Mamalis N. Endophthalmitis. J Cataract Refract Surg 2002; 28: 729-730.

2. Brinton GS, Topping TM, Hyndiuk RA, Aaberg TM, Reeser FH, Abrams GW. Posttraumatic endophthalmitis. Arch Ophthalmol 1984; 102: 547-550.

3. Essex RW, Yi Q, Charles PG, Allen PJ. Post-traumatic endophthalmitis. Ophthalmology 2004; 111: 2015-2022.

4. Thompson JT, Parver LM, Enger CL, Mieler WF, Liggett PE. Infectious endophthalmitis after penetrating injuries with retained intraocular foreign bodies. National Eye Trauma System. Ophthalmology 1993; 100: 1468-1484.

5. Kernt M, Kampik A. Endophthalmitis: Pathogenesis, clinical presentation, management, and perspectives. Clin Ophthalmol 2010; 4: 121-135.

6. Kuhn F. Urazy oka. Czelej, Lublin 2011.

7. Chun DW, Colyer MH, Wroblewski KJ. Visual and anatomic outcomes of vitrectomy with temporary keratoprosthesis or endoscopy in ocular trauma with opaque cornea. Ophthalmic Surg Lasers Imaging 2012; 43: $302-310$.

8. Barry P, Behrens-Baumann W, Pleyer U, Seal D (ed.). ESCRS Guidelines on prevention, investigation and management of post-operative endophthalmitis. European Society for Cataract \& Refractive Surgeons 2007.

9. Ashok N. Post traumatic endophthalmitis. Kerala J Ophthalmol 2010; 22: 258-261. 